\title{
AGENESIA PULMONAR SEGMENTARIA EN UN PACIENTE PEDIÁTRICO: REPORTE dE CASO CLÍNICO
}

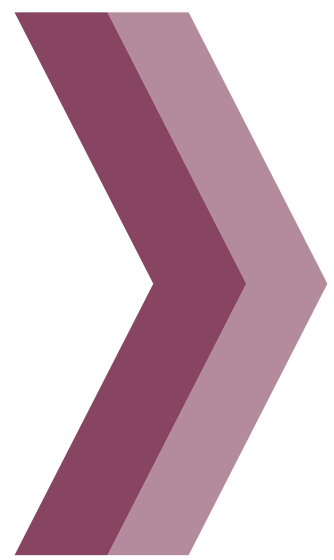

\section{PULMONARY SEGMENTARY AGENESIA IN A PEDIATRIC PATIENT: CLINICAL CASE REPORT}

\section{Arturo Recabarren Lozada ${ }^{1}$ Lizeth Yuliana Cabanillas Burgos ${ }^{2}$}

1. Neumólogo - Pediatra. Hospital III Yanahuara- EsSalud Arequipa, Docente Principal de Pediatría de la Universidad Nacional de San Agustín. Arequipa - Perú.

2. Residente de UCI Pediátrica, Hospital Base Carlos Alberto Seguín Escobedo - EsSalud. Arequipa, Perú.

\section{RESUMEN}

La agenesia pulmonar es un raro trastorno del desarrollo pulmonar. Se caracteriza por la ausencia de un bronquio principal, ausencia de pulmón y arteria pulmonar. Generalmente se asocia con otros defectos congénitos, y debe sospecharse ante la opacidad del hemitórax. Se presenta el caso de un lactante de 3 meses de edad con antecedente de prematuridad y con dependencia de oxígeno en cuya radiografía se observó radiopacidad en ápice del hemitórax derecho, y se completó el diagnóstico de agenesia pulmonar con Gammagrafía pulmonar y Angiotem pulmonar. No se encontró otras malformaciones asociadas, con una evolución favorable fue dado de alta a los 4 meses de hospitalizado sin necesidad de oxígeno y con buena ganancia ponderal. Conclusiones: La agenesia pulmonar es una entidad infrecuente. Debido a la variabilidad en la presentación clínica debe tenerse un alto índice de sospecha ante el hallazgo de la radiopacidad del hemitórax. Los métodos diagnósticos que se utilizan son radiografía, tomografía y gammagrafía. El tratamiento consiste de medidas de soporte, como oxigenoterapia, rehabilitación, prevención y tratamiento precoz de infecciones.

PALABRAS CLAVE: Agenesia pulmonar, malformación pulmonar, anomalía del desarrollo.

\begin{abstract}
Pulmonary agenesis is a rare disorder of lung development. It is characterized by the absence of a main bronchus, absence of lung and pulmonary artery. It is usually associated with other congenital defects, and should be suspected due to the opacity of the hemithorax. We present the case of a 3-month-old infant with a history of prematurity and oxygen dependence whose radiography showed radiopacity at the apex of the right hemithorax, and the diagnosis of pulmonary agenesis was completed with pulmonary gammagraphy and pulmonary angiotem. No other associated malformations were found, with a favorable evolution was discharged after 4 months of hospitalization without oxygen and with good weight gain. Conclusions: Pulmonary agenesis is an infrequent entity. Due to the variability in the clinical presentation, a high index of suspicion must be observed before the radiopacity of the hemithorax is found. The diagnostic methods used are radiography, tomography and scintigraphy. The treatment consists of support measures, such as oxygen therapy, rehabilitation, prevention and early treatment of infections.
\end{abstract}

KEYWORDS : Pulmonary agenesis, pulmonary malformation, developmental anomaly. 


\section{INTRODUCCIÓN}

La agenesia pulmonar se caracteriza por la ausencia del tejido pulmonary de estructuras bronquiales, se da por interrupción del desarrollo de la cuarta o quinta semana de gestación. No es una patología frecuente, estadísticamente la incidencia de es de 1 por 10000 a 15000 autopsias, o una prevalencia de 0.0034 a $0.0097 \%{ }^{1}$. La agenesia pulmonar es la ausencia total del parénquima pulmonar, estructuras vasculares y/o el bronquio más allá de la bifurcación ${ }^{2}$. Las manifestaciones clínicas dependen básicamente de las anomalías presentes o de complicaciones, como neumonía, bronquiolitis, asma entre otras de la vía respiratoria inferior ${ }^{3}$. Dentro de las malformaciones asociadas en la agenesia pulmonar, $37.5 \%$ son cardiovasculares, como la persistencia del conducto arterioso y el foramen oval ${ }^{3}$; también pueden presentarse otro tipo de anomalías como atresia duodenal, fístula traqueoesofágica, malformación renales y faciales ${ }^{3}$. Dichas malformaciones congénitas del pulmón y las vías aéreas son un amplio grupo de anomalías del desarrollo algunas de las cuales pueden permanecer asintomáticas hasta que se descubren de manera accidentalmente en estudios de imagen ${ }^{4}$. A pesar de no existir un pulmón el paciente puede permanecer asintomático por mucho tiempo hasta su diagnostıco ${ }^{5}$. Fue descrita por primera vez por De Pozze en 1673 quien la descubrió de manera accidental en la autopsia de una mujer adulta ${ }^{6}$. En la literatura médica hay reportados aproximadamente de 200 a 300 casos como anomalía aislada en un pequeño porcentaje, en el resto de los casos se presenta dentro de un conjunto de malformaciones complejas o asociada a malformaciones ipsilateral.

\section{CASO CLÍNICO}

Paciente varón de 3 meses de edad, producto de segundo embarazo, gestación doble; de madre de 30 años de edad con antecedente de vulvovaginitis en el III trimestre de gestación. No recibió hierro ni ácido fólico. Se le realizaron dos ultrasonidos prenatales que se reportaron normales. El niño nació por vía vaginal a las 27 semanas de gestación, Apgar 4 al minuto y 6 a los 5 minutos; peso al nacer $1100 \mathrm{~g}$, talla $40 \mathrm{~cm}$, con pobre esfuerzo respiratorio por lo que requirió intubación endotraqueal y ventilación a presión positiva. Su gemelo falleció a la semana de vida por sepsis neonatal. Durante 3 meses se le hospitalizó en la unidad de cuidados intensivos del hospital Carlos Alberto Seguín por presentar dificultad respiratoria; requirió ventilación mecánica durante 45 días, presentando como complicaciones de su prematurez y estancia hospitalaria sepsis neonatal y retinopatía del prematuro con evolución favorable. A las 39 semanas de edad corregida fue transferido al hospital Yanahuara para evaluación por Neumología Pediátrica y destete de oxígeno por displasia broncopulmonar. A su ingreso tenía Peso: 3500 gr., Talla: $50 \mathrm{~cm}$, PC: $36 \mathrm{~cm}$, FC $144 x^{\prime}$, FR: $38 x^{\prime}$, SatO2: $90-92 \%$ con 0.5 It de 02 suplementario; sin dificultad respiratoria, con lactancia materna a libre demanda; al examen se observó tórax asimétrico por disminución del diámetro anteroposterior del hemitórax izquierdo, con disminución de los movimientos respiratorios ipsilaterales. A la auscultación, ausencia de ruidos agregados y buen pasaje de murmullo vesicular. Contaba con Gasometría arterial con pH: 7.37, pO2:70 $\mathrm{mmHg}$, pCO2: $50 \mathrm{mmHg}$, HCO3:28.9 mEq/l, Na: $132 \mathrm{mEq} / \mathrm{l}, \mathrm{K}: 5.4 \mathrm{mEq} / \mathrm{l}$. La radiografía de tórax mostró radiopacidad apical en hemitórax derecho (Imagen 1); por lo que se sospechó una probable malformación pulmonar (hipoplasia vs agenesia); debido a ello se le realizó tomografía de tórax con ventana pulmonar en la que se reveló ausencia de lóbulo superior y medio de pulmón derecho y sobredistensión del pulmón izquierdo con herniación hacia el lado derecho(Imagen 2). Posteriormente se le realizó Gammagrafía de perfusión que demostró la ausencia de perfusión en el lóbulo superior y medio derechos no pudiéndose hacer la fase de ventilación debido a no contar con la colaboración del paciente por la edad. (Imagen 3). Finalmente se realizó Angiotem Pulmonar, la misma que reveló corazón de tamaño y morfología conservadas; no obstante desplazado hacia la derecha, con origen de arteria pulmonar y aorta sin alteraciones visibles; arteria pulmonar de morfología y calibre conservada, sin signos de Tromboembolismo pulmonar ni alteraciones en ramas lobares, no lográndose identificar arteria para el lóbulo superior derecho, igualmente no se identifican bronquio para este lóbulo; infiltrado en vidrio deslustrado en ambos pulmones con áreas parchada de atelectasia que sugieren displasia broncopulmonar incipiente en relación a su prematuridad(Imagen 4). Se le realizó además ecocardiografía y ecografía abdominal en busca de malformaciones asociadas, no encontrándose ninguna malformación concomitante; fue dado de alta a los 30 días de hospitalización sin necesidad de oxigenoterapia, con buena ganancia ponderal y sin patología respiratoria aguda; con indicación de controles periódicos por Neumología Pediátrica, Oftalmología y Niño de Alto Riesgo. 


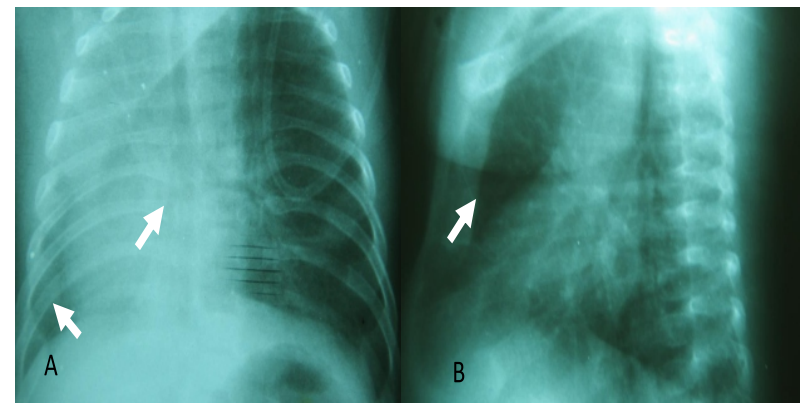

Figura 1.A. Radiografía de tórax en proyección anteroposterior se aprecia radiopacidad total de hemitórax derecho y parénquima pulmonar de lóbulo inferior derecho visible, pulmón izquierdo hiperinsuflado y herniado hacia lado derecho. B. En proyección lateral silueta cardiaca y mediastino desplazados hacia atrás por hiperinsuflación pulmonar izquierda

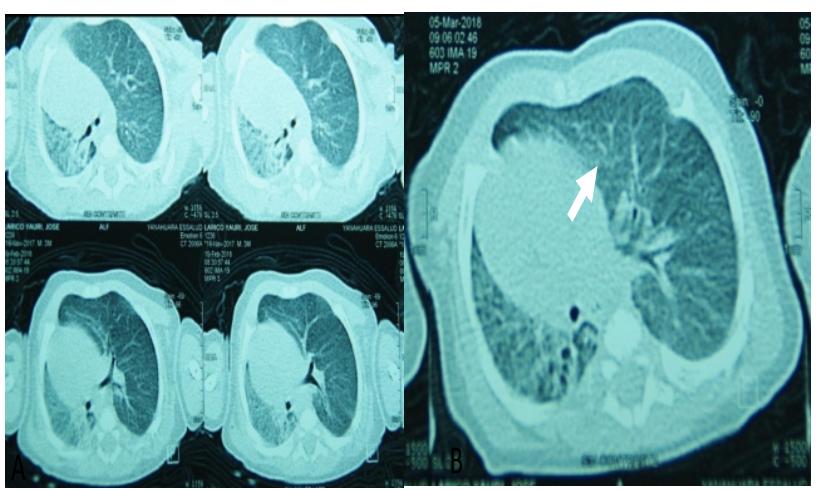

Figura 2.A. Tomografía de tórax con ventana para pulmón en la que se reveló ausencia de lóbulo superior y medio de pulmón derecho y sobredistensión del pulmón izquierdo. B. Imágenes de microatelectasias en lóbulo inferior derecho compatibles con Displasia Broncopulmonar.

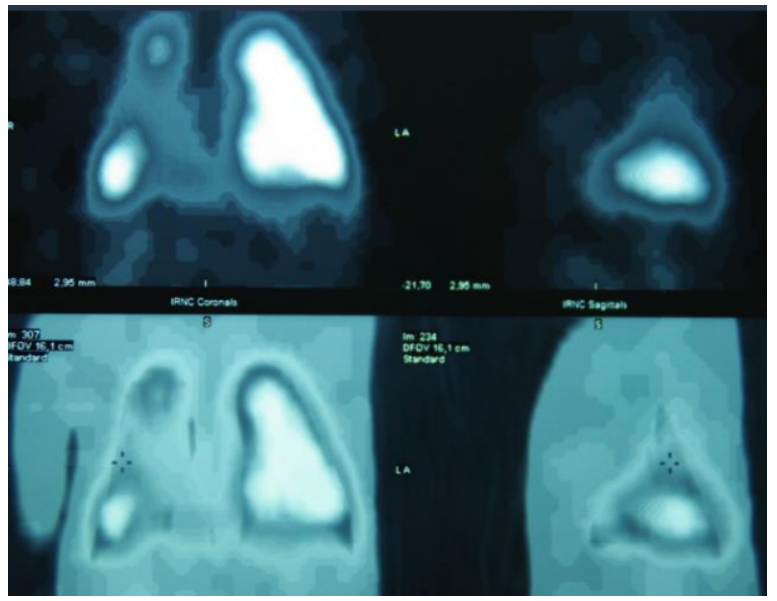

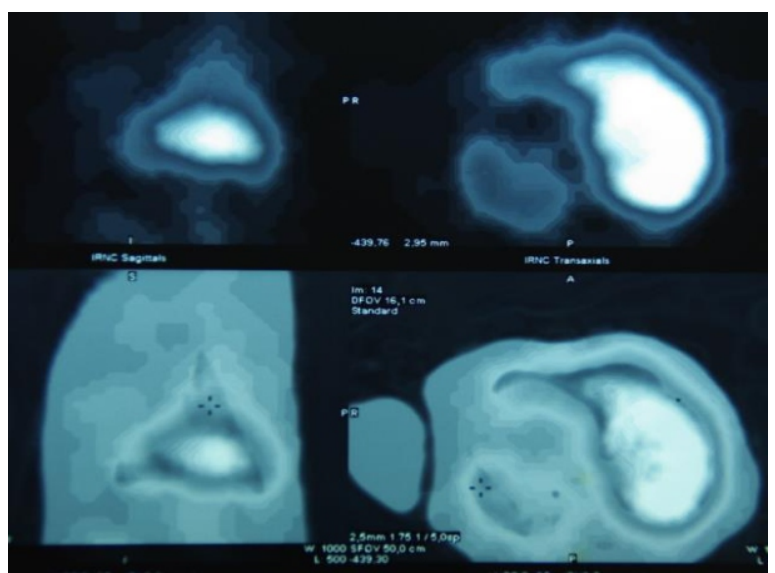

Figura 3. Gammagrafía de perfusión que muestra la falta de captación del radiotrazador en fase perfusoria en lóbulo medio y superior derechos.

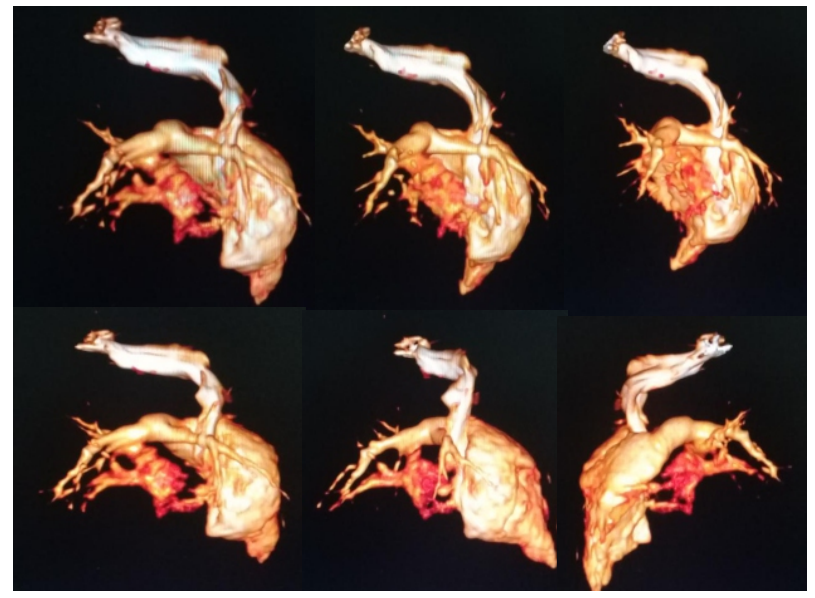

Figura 4. Angiotem Pulmonar: arteria pulmonar de morfología y calibre conservada, sin signos de TEP ni alteraciones en ramas lobares, no se logra identificar arteria para el lóbulo superior derecho, igualmente no se identifica bronquio para este lóbulo.

\section{DISCUSIÓN}

La agenesia pulmonar es una rara patología congénita incluida dentro de los trastornos del desarrollo pulmonar. Está definida por la ausencia de un bronquio principal aunado a la ausencia total del pulmón y arteria pulmonar ipsilateral $\left.\right|^{2}$. Fue descrita por primera vez en 1673 por De Pozze, quien la observó de manera incidental durante la autopsia de una joven ${ }^{6}$. El primer reporte de caso fue hecho en 1887 por Haberlein. Desde entonces, cerca de 200 casos han sido mencionados en la literatura ${ }^{7,8}$. La incidencia conjunta de aplasia y agenesia pulmonar se estima del 0.0034$0.0097 \%{ }^{1}$. Pueden ser unilaterales o bilaterales 
(incompatible con la vida), sin predominio de género ni predilección por un pulmón u otro.

La etiología subyacente es desconocida; aunque se han involucrado factores mecánicos, teratogénicos y genéticos (infección vírica, déficit de vitamina A) y su patogenia es secundaria a una alteración vascular en el desarrollo pulmonar fetal. En la mitad de los casos la agenesia pulmonar se encuentra asociada con otras malformaciones congénitas, principalmente esqueléticas, genitourinarias y gastrointestinales ${ }^{3}$.

Existen varias clasificaciones de la agenesia pulmonar unilateral, considerando que en el caso bilateral es incompatible con la vida. En 1955 Boyden la clasificó en 3 grupos: Pulmón y arteria ausentes; pulmón y arteria ausentes con un bronquio rudimentario emergiendo de la tráquea; y pulmón hipoplásico con un bronquio completamente formado.

Existe otra clasificación propuesta por Spencer también con tres grupos: ${ }^{9,10}$ Agenesia bilateral congénita completa, Agenesia unilateral y Agenesia lobar.

Schneider y Schwalbe la clasifican en otros tres grupos en el llamado complejo agenesiahipoplasia: ${ }^{9,10,11}$ Agenesia pulmonar: ausencia total de pulmón, bronquio y arteria pulmonar, Aplasia pulmonar: ausencia total de pulmón y arteria pulmonar con un bronquio principal rudimentario, Hipoplasia pulmonar: bronquio y arteria pulmonar hipoplásicos con una cantidad variable de tejido pulmonar, primaria: sin causa identificable; y secundaria: limitación del desarrollo normal del pulmón fetal (oligohidramnios, malformaciones torácicas, hernias diafragmáticas, masas pulmonares, etc.).

En el caso presentado se encontraría en el I grupo según Boyden; el III grupo de Spencer y dentro de la primera categoría de Schneider y Schwalbe.

La edad y forma de presentación es muy variable. La mayoría de los pacientes inicia con sintomatología durante el primer año de vida. Los hallazgos clínicos son igualmente escasos; se describe disminución del murmullo vesicular del lado afectado y cambios percutorios, en particular en el área axilar e infraxilar. La ventilación en el pulmón contralateral suele ser normal, al igual que la apariencia de la caja torácica. Por otro lado, hay reportes de diagnóstico en pacientes adultos sin síntomas respiratorios o diagnósticos post mórtem. La pobreza semiológica del cuadro hace que el diagnóstico descanse en gran parte en la imagenología.

Los estudios radiológicos muestran datos similares tanto en aplasia pulmonar como en la agenesia, excepto por la presencia de bronquio principal rudimentario en la aplasia. ${ }^{1,7}$. El pulmón sano generalmente presenta sobredistensión y herniación hacia el hemitórax contralateral. La tomografía computarizada ayuda a demostrar la ausencia de parénquima y arteria pulmonar ${ }^{4,12}$, así como la presencia de un bronquio rudimentario. La broncoscopía muestra la presencia de un bronquio principal terminado en un saco ciego, confirmando el diagnóstico en caso de aplasia pulmonar. Otros estudios, como la gammagrafía pulmonar perfusoria/ventilatoria, son útiles para distinguir la ausencia de pulmón. Sin embargo, este estudio no diferencia entre aplasia y agenesia. El pronóstico depende de múltiples factores entre los que destacan malformaciones congénitas coexistentes, infección del pulmón único, hipertensión arterial pulmonar y acodamiento de los grandes vasos debido al desplazamiento mediastinal.

La mortalidad es del $33 \%$ durante el primer año de vida y del $50 \%$ en los primeros 5 años $^{2}$. Sin embargo, si un paciente sobrevive los primeros 5 años de vida, se puede esperar una vida normal sin mayores complicaciones ${ }^{3,13}$. El tratamiento consiste de medidas de soporte, como oxigenoterapia, rehabilitación (para mejorar el aclaramiento de secreciones), prevención y tratamiento precoz de infecciones; en algunos casos, la elevación quirúrgica del hemidiafragma ipsilateral al defecto ha mostrado buenos resultados. En los casos de evolución tórpida debe realizarse la escisión quirúrgica del muñón. ${ }^{13}$

En conclusión, se trata del caso de una agenesia pulmonar segmentaria en un lactante prematuro, que presentaba una opacidad en hemitórax derecho, asintomático y en la que los estudios complementarios de imagenología corroboraron el diagnóstico planteado. 


\section{REFERENCIAS BIBLIOGRÁFICAS}

1. Vargas S, Peñaloza C, De Caro A, Arispe E, Acuña M (2009) Agenesia pulmonar asociada a persistencia del conducto arterioso y su tratamiento percutáneo; a propósito de un caso. Avances Cardiol 29: 377-379.

2. Uzcátegui N, Simancas F, Zarate M, Jáuregui Y (2013) Hallazgo casual de Agenesia Pulmonar derecha asociada a Ano Imperforado: Reporte de un caso. Revmédcient 26: 2329.

3. Bolaños-Jiménez R, Rivera-Silva G, Treviño-Alanís Ma, Moreno-Treviño Ma (2011) Agenesia del Pulmón Derecho en Recién Nacida. Bol ClinHospInfant Edo Son 28: 59-60.

4. Hermoso-TorregrosaC, Moreno-Medinilla E,Pérez Ruiz E, Caro Aguilera P, Pérez Frías FJ (2014) Hiperinsuflación lobar congénita: manejo conservador como alternativa terapéutica.AnPediatr (Barc) 81: 45-48.

5. Margarita AR (2001) Malformaciones pulmonares congénitas. Revchilpediatr 72:52-57.

6. Rosenberg DH (1962) Pulmonaryagenesis 42: 68-73.

7. Calvo G Mario, Krause H Sergio, Horzella R Roberto, Sanchez H Alfonso, Jiménez P Patricio (1983) Agenesia Pulmonar. Revchilpediatr 54:261-265.

8. Alvarez JA, Vaccaro UMI, Verdejo PH, Villarroel QC, Puentes
RR (2000) Agenesia pulmonar unilateral con malformaciones múltiples: reporte de un caso. Revchilpediatr 71:41-45.

9. Manohar VM, Shivanna DN, Ramesh, Vemgal P. Left pulmonary agenesis with single atrium simulating cardiac type of total anomalous pulmonary venous connection. Ind J ThoracCardiovascSurg 2008;24;180-183.

10. Pavlovic V, Stojanovic V, Doronjski A, Kovacevic B. Unilateral Agenesis of Lung Associated with Total Anomalous Pulmonary Venous Return and Atrial Septal Defect. Cent Eur J Med 2011;6(3);353-355.

11. Managoli S, Chaturvedi P, Vilhekar KY, Gagane N. Unilateral Pulmonary Agenesis and Renal Anomalies Associated with in Situ Neuroblastoma of the Adrenal Gland. Indian J Pediatr 2004;71(6);545-547.

12. Bhagat R, Panchal N, Shah A. Pulmonary aplasia: a CT appearance. Indian Pediatr. 1992; 29:1410-2.

13. Krivchenya DU, Rudenko EO, Lysak SV, Dubrovin AG, Khursin VN, Krivchenya TD. Lung aplasia: anatomy, history, diagnosis and surgical management. Eur J Pediatr Surg. 2007; 17:24450.

\section{Correspondencia}

Arturo Recabarren Lozada

arturorecabarren@gmail.com
Fecha de recepción: 11 de mayo de 2018

Fecha de aceptación: 10 de junio de 2018 